\title{
Low Order Modes of an Ion Cloud in a Penning Trap*
}

\author{
J. J. Bollinger, D. J. Heinzen, † F. L. Moore, W. M. Itano and D. J. Wineland
}

Time and Frequency Division, National Institute of Standards and Technology, 325 Broadway, Boulder, Colorado 80303, U.S.A.

Received November 1, 1991; accepted March 26, 1992

\begin{abstract}
The electrostatic modes of a cloud of ions confined in a Penning trap are discussed in the limit that the Debye length is small compared to the cloud dimensions and the cloud dimensions are small compared to the trap dimensions. Experimental measurements of some of these mode frequencies on plasmas of laser-cooled $\mathrm{Be}^{+}$ions agree well with calculations. Observation of the modes provides a nondestructive method for obtaining information on the ion density and cloud shape. In addition, excitation of the modes by static field asymmetries may provide a practical limit to the density and number of charged particles that can be stored in a Penning trap.
\end{abstract}

A cloud of charged particles stored in a Penning trap can be considered a plasma, in particular a nonneutral plasma, under conditions where the Debye length $\lambda_{D}$ is small compared to the cloud dimensions $[1,2]$. The Debye length is given by

$\lambda_{\mathrm{D}}^{2}=\frac{k_{\mathrm{B}} T}{4 \pi n_{0} q^{2}}$

where $k_{\mathrm{B}}$ is Boltzmann's constant, $T$ is the plasma temperature, $n_{0}$ is the plasma density, and $q$ is the particle charge. In this limit the density is constant in the plasma interior and drops to 0 at the plasma boundary over a distance on the order of a few Debye lengths $[2,3]$. The density is determined by the rotation frequency $\omega$ of the plasma about the trap axis by

$n_{0}=\frac{m \omega(\Omega-\omega)}{2 \pi q^{2}}$.

Here $m$ is the mass of a particle and $\Omega \equiv q B /(m c)$ is the cyclotron frequency where $B$ is the magnetic field and $c$ is the speed of light. If the plasma dimensions are small compared to the trap dimensions, the electrostatic potential $\phi_{\mathrm{T}}$ of the trap may be assumed to be quadratic,

$\phi_{\mathrm{T}}=\frac{m \omega_{z}^{2}}{4 q}\left(2 z^{2}-r^{2}\right)$,

and the effect of image charges in the trap electrodes neglected. Here $r$ and $z$ are cylindrical coordinates and $\omega_{z}$ is the single particle axial frequency. When all of the above conditions are satisfied $\left[\lambda_{\mathrm{D}} \ll\right.$ plasma dimensons, $\phi_{\mathrm{T}}$ is given by eq. (3), and image charges can be neglected], the plasma has the simple shape of a spheroid (an ellipsoid of revolution) [4]. The aspect ratio of the spheroid (the ratio of

\footnotetext{
* Contribution of the U.S. Government, not subject to copyright.

$\dagger$ Present address: Physics Department, University of Texas, Austin, TX 78712 , U.S.A.
}

the plasma axial extent to the plasma radial diameter) is also determined by the plasma rotation frequency $\omega$ [4]. Constant density equilibria exist for $\omega_{\mathrm{m}}<\omega<\Omega-\omega_{\mathrm{m}}$ where $\omega_{\mathrm{m}}$ is the single particle magnetron frequency. For $\omega$ slightly greater than $\omega_{\mathrm{m}}$, the plasma is shaped like a pancake. As $\omega$ increases, the plasma density increases and the plasma aspect ratio increases by decreasing the plasma radius and increasing the plasma axial extent. At $\omega=\Omega / 2$, the plasma obtains its maximum density and maximum aspect ratio. The condition $\omega=\Omega / 2$ is often referred to as Brillouin flow. At Brillouin flow the plasma behaves in many ways like an unmagnetized plasma [5]. As $\omega$ increases beyond $\Omega / 2$, the plasma aspect ratio and density decrease. Spheroidal plasmas in a Penning trap are also discussed in Ref. [6].

A surprising result is that analytic solutions exist for all of the electrostatic modes of a cold, spheroidal nonneutral plasma in a uniform magnetic field (a Penning trap plasma) [7-9]. Some of the low order mode frequencies have been measured on plasmas of laser-cooled ${ }^{9} \mathrm{Be}^{+}$ions and are in good agreement with the calculations [8]. This paper gives a brief description of the low order modes and the measurements on the ${ }^{9} \mathrm{Be}^{+}$plasmas. More details can be obtained in Refs [7-9]. These modes may be important for a number of Penning trap experiments. For example, observation of the modes can provide a nondestructive method for obtaining information on the plasma density and shape when other imaging techniques are not available. This is the case for antimatter $[10,11]$ and electron plasmas. In addition, some of the plasma modes are zero-frequency modes for particular plasma densities. Field errors in the trapping potential can excite these modes and enhance radial transport [12, 13]. Excitation of these modes may therefore set a practical limit on the density and number of particles that can be stored in a Penning trap.

The electrostatic modes of a Penning trap plasma can be described, with the use of spheroidal coordinates, by two integers $(l, m)$ with $l \geqslant 1$ and $m \geqslant 0$ [7]. (Negative values of $m$ are allowed, but do not give rise to new modes.) The index $m$ indicates that the plasma mode displays an $\exp (i m \phi)$ dependence where $\phi$ is the azimuthal angle. The index $l$ describes the mode dependence along a spheroidal surface (for example, the plasma boundary) in a direction perpendicular to $\hat{\phi}$. The $l=1$ modes are the familiar centerof-mass modes where the ion plasma keeps its same shape, but the center of mass of the plasma executes one of the three motions of a single ion in a Penning trap. For example, the $(1,0)$ mode is the axial center-of-mass mode at frequency $\omega_{z}$. There are two $(1,1)$ modes which correspond 
to the perturbed cyclotron and magnetron center of mass modes at frequencies $\Omega-\omega_{\mathrm{m}}$ and $\omega_{m}$. These center-of-mass frequencies can, in general, be measured or calculated very precisely.

Excitation of an $l=2$ mode produces a quadrupole deformation of a plasma in a Penning trap. For example, when a $(2,0)$ mode is excited, the plasma always stays spheroidal, but the aspect ratio of the spheroid oscillates in time at the $(2,0)$ mode frequency $\omega_{20}$. There are two $(2,0)$ modes: a low frequency mode with a frequency $\omega_{20}^{-}$on the order of the trap axial frequency $\omega_{z}$ and a high frequency mode with a frequency $\omega_{20}^{+}$on the order of the cyclotron frequency $\Omega$. The low frequency mode is called a plasma mode. In this mode, the axial and radial excursions of the plasma are $180^{\circ}$ out of phase. The high frequency mode is called an upper hybrid mode. In this mode the axial and radial excursions of the plasma are in phase. There are three $(2,1)$ modes. In each of these modes the plasma keeps its equilibrium shape (for small amplitudes of excitation) but is tilted (or rotated) with respect to the magnetic field axis ( $z$ axis) of the trap. The tilted plasma then precesses about the magnetic field axis at the mode frequency. In a frame of reference rotating with the plasma, one of these modes precesses in a direction opposite to the plasma rotation and for a particular rotation frequency can be a zero frequency mode in the lab frame. This zero-frequency mode can be excited by a static field error $[12,13]$. For example, it can be excited by a tilt of the magnetic field with respect to the electrode symmetry axis. There are two $(2,2)$ modes. In both of these modes, the plasma becomes an ellipsoid with unequal principal axes in the $z=0$ plane. The ellipsoid rotates about the $z$-axis at the $(2,2)$ mode frequency.

Most Penning trap experiments have been done with the plasma rotation frequency $\omega \ll \Omega$. In the experiment of Ref. [8], we used radiation pressure from two lasers to remove energy and control the angular momentum of a plasma of ${ }^{9} \mathrm{Be}^{+}$ions $[8,14]$. This enabled us to obtain rotation frequencies throughout the allowed range $\omega_{\mathrm{m}}<\omega<\Omega-\omega_{\mathrm{m}}$. The static $(2,1)$ mode was excited on a plasma of $2000{ }^{9} \mathrm{Be}^{+}$ ions with $B=0.82 \mathrm{~T}\left[\Omega\left({ }^{9} \mathrm{Be}^{+}\right) /(2 \pi)=1.4 \mathrm{MHz}\right]$. This mode was excited by a tilt of the magnetic field when the plasma rotation frequency was increased to the rotation frequency where the $(2,1)$ mode is static in the lab frame. Excitation of this mode was detected by a change in the ion fluorescence produced by an accompanying heating of the ion plasma. Figure 1 shows the measured rotation frequency where heating was observed as a function of $\omega_{z}$. Good agreement is obtained with the calculated rotation frequency $\omega$ at which $\omega_{21}=0$. For $2000{ }^{9} \mathrm{Be}^{+}$ions, heating from the excitation of this static mode was observed for misalignments of the trap magnetic and electric field axes of greater than $0.01^{\circ}$. We have also been able to spin up plasmas consisting of $40000{ }^{9} \mathrm{Be}^{+}$ions at $B=6 \mathrm{~T}\left[\Omega\left({ }^{9} \mathrm{Be}^{+}\right) /(2 \pi)=10.2 \mathrm{MHz}\right]$. With care we could obtain ion densities near $10^{10} \mathrm{~cm}^{-3}$ when $\omega \simeq \Omega / 2$. For this larger number of ions with $B=6 \mathrm{~T}$, the $(2,1)$ heating resonance was much stronger. In addition, we observed other heating resonances at rotation frequencies less than the $(2,1)$ heating resonance. These additional resonances presumably correspond to higher order static resonances which have recently been calculated by Dan Dubin of the University of California at San Diego. They tend to limit the plasma density to even lower values

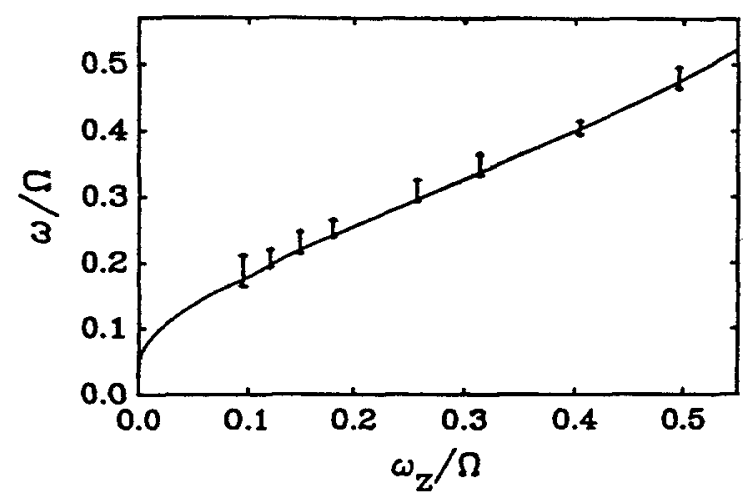

Fig. 1. Plasma rotation frequency $\omega$ at which heating was observed as a function of the single-particle axial frequency $\omega_{z}$. Both frequencies are expressed in units of the cyclotron frequency $\Omega$. The solid line indicates the calculated rotation frequency $\omega$ at which $\omega_{21}=0$. This is a universal curve for any charged particle species involving no adjustable parameters

than the static $(2,1)$ resonance. This has important implications for work where high densities and large numbers of charged particles are to be stored in a Penning trap.

We have also detected and measured the $(2,0)$ plasma and upper hybrid modes on plasmas of a few thousand ${ }^{9} \mathrm{Be}^{+}$ions at $B=0.82 \mathrm{~T}$. These modes were excited by applying a potential with a sinusoidal time dependence between the ring and endcap electrodes of the trap. They were detected by a change in the ion fluorescence when the frequency of the applied of was resonant with the mode frequency. Measurements of the plasma mode frequency as a function of the plasma rotation frequency are shown in Fig. 2 for two different trap axial frequencies. The solid line shows the calculated plasma mode frequency. Good agreement between the predicted and observed modes is obtained with no adjustable parameters. Figure 2 shows two additional calculations. In the first, shown as dashed lines, the magnetic field is assumed to be effectively infinite; that is, the ions are not allowed to move radially, and the mode frequency is calculated assuming a simple axial stretch of the charged spheroid. In the second calculation, shown as dotted lines, the magnetic field is assumed to be effectively 0 . The $(2,0)$ plasma mode behaves like a mode of a strongly magnetized plasma at low rotation frequencies and an

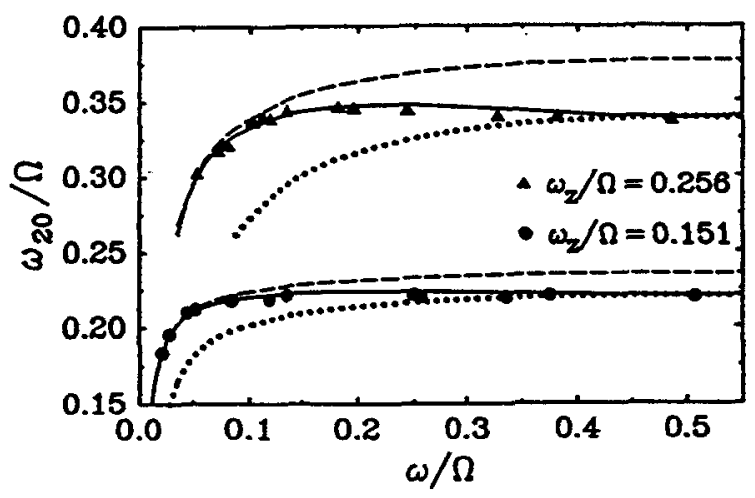

Fig. 2. $(2,0)$ plasma mode frequency $\omega_{20}^{-}$as a function of the rotation frequency $\omega$ for $\omega_{z} / \Omega=0.151$ and $\omega_{z} / \Omega=0.256$. All frequencies are expressed in units of the cyclotron frequency $\Omega$. The theoretical curves are therefore independent of the particle change-to-mass ratio. The circles and triangles give the experimental data. The solid lines give the cold-fluid model predictions for $\omega_{20}^{-}$. The dashed and dotted lines give the high- and lowmagnetic-field calculations for $\omega_{20}^{-}$, respectively 


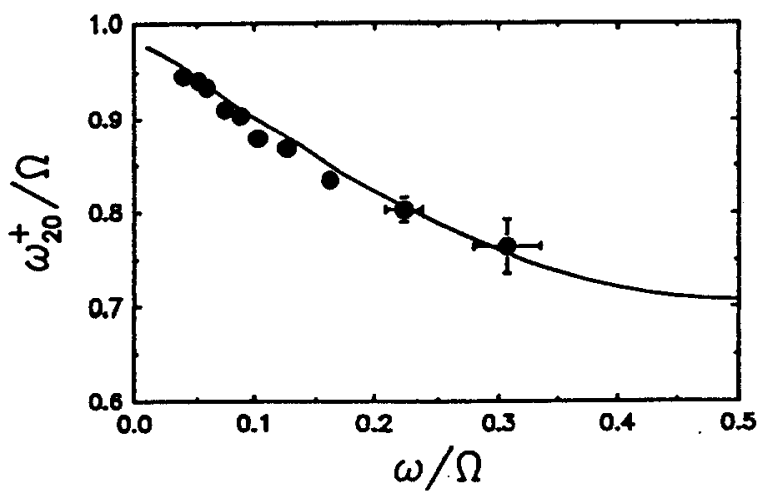

Fig. 3. Upper hybrid mode frequency $\omega_{20}^{+}$as a function of the rotation frequency $\omega$ for $\omega_{z} / \Omega=0.151$. All frequencies are expressed in units of the cyclotron frequency. The circles give the experimental data. The uncertainty for measurements done at low rotation frequencies is approximately the size of the circles. At high rotation frequencies the uncertainties increase because the mode becomes difficult to excite. The solid line gives the coldfluid model predictions

unmagnetized plasma near the Brillouin limit. Figure 3 shows measurements of the upper hybrid $(2,0)$ mode along with calculations of this mode frequency. We could not excite this mode near the Brillouin limit because the mode becomes difficult to couple to with external fields there [9]. Measurement of either the plasma or the upper hybrid $(2,0)$ mode determines the plasma rotation frequency and aspect ratio. Therefore measurement of these modes may be useful in experiments with trapped positrons [10] and antiprotons [11] where other nondestructive means of obtaining this information are not available. We have done some calculations which provide the plasma rotation frequency (and therefore the density and aspect ratio) for a measured $(2,0)$ mode frequency. These will be included in a future publication [9].

We gratefully acknowledge the support of the Office of Naval Research. We thank S. Gilbert and C. Weimer for their suggestions and careful reading of the manuscript.

\section{References}

1. Malmberg, J. H. and de Grassie, J. S., Phys. Rev. Lett. 35, 577 (1975).

2. Driscoll, C. F., Malmberg, J. H. and Fine, K. S., Phys. Rev. Lett. 60, 1290 (1988).

3. Prasad, S. A. and O'Neil, T. M., Phys. Fluids 22, 278 (1979).

4. Brewer, L. R., Prestage, J. D., Bollinger, J. J., Itano, W. M., Larson, D. J. and Wineland, D. J., Phys. Rev. A38, 859 (1988).

5. Davidson, R. C., in: "Non-neutral Plasma Physics" (Edited by C. W. Roberson and C. F. Driscoll) (AIP Conf. Proc. No. 175) (American Institute of Physics, New York 1988), pp. 139-208.

6. Jeffries, J. B., Barlow, S. E. and Dunn, G. H., Int. J. Mass Spectrom. Ion Processes 54, 169 (1983).

7. Dubin, D. H. E., Phys. Rev. Lett. 66, 2076 (1991).

8. Heinzen, D. J., Bollinger, J. J., Moore, F. L., Itano, W. M. and Wineland, D. J., Phys. Rev. Lett. 66, 2080 (1991).

9. Bollinger, J. J., Heinzen, D. J., Moore, F. L., Itano, W. M., Wineland, D. J. and Dubin, D. H. E., manuscript to be submitted to Phys. Rev. A.

10. Surko, C. M., Leventhal, M. and Passner, A., Phys. Rev. Lett. 62, 901 (1989).

11. Gabrielse, G., Fei, X., Orozco, L. A., Tjoelker, R. L., Hass, J., Kalinowsky, H., Trainor, T. A. and Kells, W., Phys. Rev. Lett. 63, 1360 (1989); 65, 1317 (1990).

12. Eggleston, D. L., O'Neil, T. M. and Malmberg, J. H., Phys. Rev. Lett. 53, 982 (1984).

13. Keinigs, R., Phys. Fluids 24, 860 (1981); 27, 1427 (1984).

14. Itano, W. M., Brewer, L. R., Larson, D. J. and Wineland, D. J., Phys. Rev. A38, 5698 (1988). 